Gynäkologe 2021 · 54:732-740

https://doi.org/10.1007/s00129-021-04829-x

Angenommen: 11. Juni 2021

Online publiziert: 8 . Juli 2021

(c) Der/die Autor(en) 2021

Redaktion

B. Sonntag, Hamburg

G. Emons, Göttingen

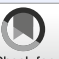

Check for
updates

\section{In diesem Beitrag}

- Kardiovaskuläre und metabolische Risikofaktoren

- Ätiologie des PCOS

- Das Mikrobiom

- Zusammenhang zwischen Darmmikrobiom und PCOS

Verminderte Diversität des Mikrobioms bei PCOS

- Insulinresistenz und Androgene

- Gallensäurenstoffwechsel

- Rolle des Trimethylamin-N-Oxid (TMAO)

- Therapieoptionen

Gewichtsreduktion - Metformin • Ballaststoffe - Probiotika - Präbiotika und andere Nahrungsergänzungsmittel

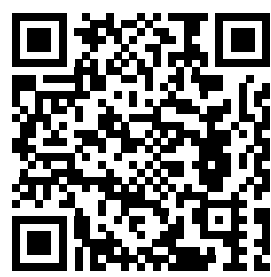

QR-Code scannen \& Beitrag online lesen

\title{
Mikrobiom und Stoffwechsel beim polyzystischen Ovarialsyndrom (PCOS)
}

\author{
Wolfgang R. Heizmann · Christoph Keck \\ Medical Affairs, Amedes MDL, Hamburg, Deutschland
}

\section{Zusammenfassung}

Das Syndrom der polyzystischen Ovarien (PCOS) gehört zu den häufigen Hormonstörungen bei Frauen in der fertilen Lebensphase. Die Ätiologie ist bis heute nicht bekannt, allerdings konnte in den letzten Jahren das Verständnis der pathophysiologischen Zusammenhänge deutlich verbessert werden. Neben endokrinologischen und metabolischen Aspekten findet der Zusammenhang zwischen PCOS und Darmmikrobiom zunehmende Beachtung. Es konnte gezeigt werden, dass das Darmmikrobiom von Frauen mit PCOS sich signifikant vom Mikrobiom nichtbetroffener Frauen unterscheidet. Daraus lassen sich therapeutische Konsequenzen ableiten, welche die Behandlung von PCOS-Patientinnen in Zukunft maßgeblich beeinflussen könnten.

\section{Schlüsselwörter}

Gastrointestinales Mikrobiom · Metabolische Erkrankungen · Endokrinologie · Fertilität · Androgene

\section{Hintergrund}

Zur Diagnose des PCOS (polyzystisches Ovarsyndrom) werden neben den Definitionen der NIH (National Institutes of Health) oder der AES (Androgen Excess Society) meist die sog. Rotterdam-Kriterien der ESHRE (European Society of Human Reproduction and Embryology) herangezogen [1]. Danach sollen mindestens 2 der 3 unten aufgeführten Kriterien vorhanden sein:

- Oligo-/Amenorrhö,

- Hyperandrogenämie/Hyperandrogenismus,

- typisches sonographisches Bild der Ovarien bei Erwachsenen.

Das PCOS ist eine der häufigsten Erkrankungen im reproduktiven Alter. Die weltweite Prävalenz liegt - in Abhängigkeit von den diagnostischen Kriterien - zwischen 5 und $12 \%$ (• Tab. 1).

Aufgrund der hohen Varianz des Phänotyps kommt es häufig zu einer verzögerten Diagnose; bis zu 70\% der betroffenen Frauen bleiben undiagnostiziert.
Je nachdem welche Symptome im Vordergrund stehen, gibt es klinisch unterschiedliche Erscheinungsformen des PCOS. Für viele Frauen führt vor allem die Hyperandrogenämie/der Hyperandrogenismus zu erheblichem Leidensdruck. Als Hyperandrogenämie wird eine vermehrte Bildung und Sekretion männlicher Hormone bei der Frau bezeichnet. Unter Hyperandrogenismus versteht man die klinischen Symptome (Hirsutismus [Ferriman-Gallwey-Score $\geq 8$ ], Akne, Effluvium bzw. androgenetische Alopezie) bei verstärkter Androgenwirkung [3]. Grundsätzlich ist ein Hyperandrogenismus auch bei normalen Androgenspiegeln möglich, sofern es trotz normwertiger AndrogenSerumkonzentration zu den entsprechenden Symptomen kommt.

\section{》) Vor allem der Hyperandro- genismus führt zu erheblichem Leidensdruck}

Hyperandrogenämie bzw. Hyperandrogenismus betreffen bis zu $78 \%$ aller Patientinnen mit PCOS [4]. Die Erfassung der bio- 
Tab. 1 Weltweite Prävalenz des PCOS [2]

\begin{tabular}{|l|l|l|l|}
\hline Kaukasierinnen & Asiatinnen & Hispanierinnen & $\begin{array}{l}\text { Afroamerika- } \\
\text { nerinnen }\end{array}$ \\
\hline 6,5-8,7\% nach NIH-Kriterien & $\begin{array}{l}2,2 \% \text { nach NIH- } \\
\text { Kriterien }\end{array}$ & $\begin{array}{l}\text { 6,0\% nach NIH- } \\
\text { Kriterien }\end{array}$ & $\begin{array}{l}3,4-8,8 \% \text { nach } \\
\text { NIH-Kriterien }\end{array}$ \\
\hline $\begin{array}{l}10,2 \% \text { nach AES-Kriterien } \\
\begin{array}{l}11,9 \% \text { nach Rotterdam-Kriteri- } \\
\text { en 2003 }\end{array}\end{array}$ & $\begin{array}{l}5,6-6,3 \% \text { nach } \\
\text { Rotterdam-Kriterien } \\
2003\end{array}$ & $\begin{array}{l}6,6 \% \text { nach Rotter- } \\
\text { dam-Kriterien 2003 }\end{array}$ & \\
\cline { 1 - 2 } $\begin{array}{l}\text { PCOS polyzystisches Ovarsyndrom, NIH National Institutes of Health (USA), AES Androgen Excess } \\
\text { Society, Rotterdam-Kriterien 2003 European Society of Human Reproduction and Embryology }\end{array}$ \\
\hline
\end{tabular}

\begin{tabular}{|c|c|}
\hline & LoE/EG \\
\hline Übergewicht/Adipositas (BMI, Hüftumfang, Kontrolle 6-12 Monate) & ++++ \\
\hline $\begin{array}{l}\text { Nikotinkonsum, Dyslipidämie, arterielle Hypertonie, gestörte Glukosetoleranz/ } \\
\text { Insulinresistenz, Bewegungsmangel }\end{array}$ & ++++ \\
\hline $\begin{array}{l}\text { Alle Übergewichtigen oder Adipösen: Nüchternlipidprofil (HDL, LDL, Cholesterin, } \\
\text { Triglyzeride) }\end{array}$ & ++++ \\
\hline Mindestens jährliche Blutdruckmessung aller Frauen mit PCOS & ++++ \\
\hline $\begin{array}{l}\text { Prävalenz von GDM, gestörter Glukosetoleranz und DM erhöht, auch bei Schlanken, } \\
\text { abhängig von der Ethnizität }\end{array}$ & ++++ \\
\hline $\begin{array}{l}\text { oGTT, Nüchternglukose oder HbA1c sollten allen Frauen mit PCOS initial als Scree- } \\
\text { ning angeboten werden }\end{array}$ & ++++ \\
\hline Präkonzeptionell $75 \mathrm{~g}$ oGTT, wenn nicht erfolgt $<20$. SSW & ++++ \\
\hline \multicolumn{2}{|c|}{$\begin{array}{l}\text { BMI Body-Mass-Index, DM Diabetes mellitus, GDM Gestationsdiabetes, } H D L \text { "high-density lipopro- } \\
\text { teins", } L D L \text { "low-density lipoproteins", LoE/EG "level of evidence"/Empfehlungsgrad, oGTT oraler } \\
\text { Glukosetoleranztest, PCOS polyzystisches Ovarsyndrom, SSW Schwangerschaftswoche(n) }\end{array}$} \\
\hline
\end{tabular}

chemischen Veränderungen und auch der klinischen Symptome ist mitunter schwierig, da zum einen die Bestimmungsmethoden zur Androgenmessung variieren, zum anderen aber auch Ethnizität, Gewicht und Alter einen Einfluss auf die Ausprägung der Symptomatik haben $[5,6]$.

Zur Messung des freien Testosterons steht bislang kein guter Assay zur Verfügung. Daher wird üblicherweise zusätzlich zur Bestimmung des Gesamttestosterons auch der Spiegel des Sexualhormon bindenden Globulins (SHBG) ermittelt, um daraus den Anteil des freien Testosterons ableiten zu können, den freien Androgenindex: $\mathrm{FAI}=100 \times($ Gesamttestosteron/SHBG) [7]. Im Idealfall sollte die biochemische Diagnostik mittels Flüssigchromatographie mit Massenspektrometriekopplung („liquid chromatography/mass spectrometry", LC/MS) als hochwertigem Analysesystem erfolgen [6]. Dies wird in der Praxis aufgrund der höheren Kosten und der geringen Verfügbarkeit jedoch nur sehr selten umgesetzt.

\section{Kardiovaskuläre und metabolische Risikofaktoren}

Neben den oben genannten Symptomen lassen sich bei vielen Frauen mit PCOS kardiovaskuläre oder metabolische Risikofaktoren nachweisen. Die internationale Leitlinie empfiehlt daher, bei allen PCOS-Patientinnen kardiovaskuläre Risikofaktoren abzuklären (z.B. BMI [Body-Mass-Index], Hüftumfang, Blutdruckmessung; - Tab. 2). Bei Übergewicht bzw. Adipositas wird zusätzlich auch ein Nüchternlipidprofil empfohlen [6].

\section{》) Auch die Bestimmung des HoMA-IR-Indexes kann bei PCOS hilfreich sein}

Unabhängig vom BMI haben Frauen mit PCOS ein erhöhtes Risiko für die Entwicklung einer Insulinresistenz mit gestörter Glukosetoleranz, Typ 2 Diabetes mellitus sowie - im Falle einer Schwangerschaft Gestationsdiabetes [8].

Die Testverfahren zum Nachweis einer Insulinresistenz weisen in der routinemäßigen Anwendung methodische Unschärfen auf. Bislang wird empfohlen, als Screening die Messung der Nüchternglukose und des $\mathrm{HbA} 1 \mathrm{c}$ zu veranlassen [6]. Zudem soll ein oraler Glukosetoleranztest (75-g-oGTT) durchgeführt werden. Bei Frauen mit PCOS kann auch die Bestimmung des HoMAIR(Homeostatic Model Assessment for Insulin Resistance)-Indexes hilfreich sein [9].

Im Falle einer Schwangerschaft sollte der oGTT noch vor der 20. Schwangerschaftswoche erfolgen, sofern zuvor kein oGTT durchgeführt wurde.

\section{Ätiologie des PCOS}

Die Ursache des PCOS ist bis heute nicht endgültig geklärt. Es gibt Hypothesen zu den pathophysiologischen Mechanismen, die zur Ausprägung des PCOS beitragen, ebenso wurden die endokrinen Veränderungen beim PCOS umfassend diskutiert. Aus der Mikrobiomforschung ergeben sich nun völlig neue Ansätze zum Verständnis der Pathophysiologie des PCOS.

Mit der Entwicklung moderner Sequenziertechniken (Next Generation Sequencing, NGS) kann die mikrobielle Besiedlung eines Habitats umfassend analysiert werden. Damit ließen in den letzten Jahren fundamental neue Erkenntnisse zur Bedeutung des Mikrobioms für die Entstehung chronischer Erkrankungen gewinnen.

\section{I) Aus der Mikrobiomforschung ergeben sich neue Ansätze zum Verständnis der Pathophysiologie des PCOS}

Die bisherigen Erkenntnisse zum Zusammenhang zwischen dem Darmmikrobiom und der Entstehung des PCOS sollen im Folgenden dargestellt werden.

\section{Das Mikrobiom}

Das Mikrobiom bezeichnet die Gesamtheit aller Mikroorganismen, die ein Lebewesen natürlicherweise - ohne Auslösung von Krankheitssymptomen - besiedeln. Der Mensch besitzt etwa $3 \times 10^{13}$ Körperzellen und beherbergt $3,8 \times 10^{13}$ bis $10^{14}$ Bakterien, davon die Mehrzahl im Darm (ca. 1011/g Faeces; $[10,11]$ ). Das metabolische Potenzial der Bakterien entspricht dem der Leber. Nach heutigem Kenntnis- 
stand enthält die Gesamtheit dieser Bakterien deutlich mehr Gene als der Mensch (ca. 9 vs. 23.000 Mio.; [12]). Neben Bakterien sind Viren einschließlich Bakteriophagen sowie Pilze natürliche Besiedler des Menschen.

Es gibt Autoren, die das Mikrobiom des Menschen als eigenes „Organ“ betrachten, dessen Organismen in einem bestimmten Gleichgewicht zueinander stehen [13]. Solange diese Homöostase aufrechterhalten wird, dient dies der Gesundheit des Menschen. Eine Verschiebung des Gleichgewichts führt zu entsprechenden Krankheitszuständen, die sich sowohl als akute als auch als chronische Erkrankung manifestieren können.

\section{Zusammenhang zwischen Darmmikrobiom und PCOS}

Tremellen und Pearce [14] wiesen erstmals auf einen möglichen Zusammenhang zwischen dem Auftreten eines PCOS und Veränderungen in der Darmflora hin. Die Autoren postulierten, dass Veränderungen des intestinalen Mikrobioms zur Auflockerung der "tight junctions" der Epithelzellen des Darms führen. Dadurch kommt es zum Einstrom von Lipopolysacchariden (LPS, Endotoxin) der Zellwand von Proteobakterien (Gram-negative Bakterien, wie z.B. Escherichia coli) mit nachfolgender Aktivierung des Immunsystems und zur Entwicklung einer niederschwelligen Inflammation mit erhöhten TNF-a und CRP(C-reaktives Protein)-Spiegeln $[15,16]$. Dies kann in weiterer Folge zur Entwicklung einer Insulinresistenz mit chronisch erhöhten Insulinspiegeln führen. Erhöhte Insulinspiegel werden als ein wesentlicher Pathomechanismus bei der Entwicklung einer Hyperandrogenämie angesehen.

\section{॥) Erhöhte Insulinspiegel sind ein Pathomechanismus bei der Entwicklung einer Hyperandrogenämie}

Das intestinale Mikrobiom ist bei Frauen und Männern in der Zusammensetzung unterschiedlich [17, 18]. Bei Männern korrelieren die relativen Anteile von Acinetobacter, Dorea, Ruminococcus und Megamonas signifikant mit dem Testosteronspiegel, während bei Frauen die relativen
Anteile von Slackia und Butyricimonas mit dem Östradiolspiegel in Zusammenhang stehen [19]. Frauen ohne PCOS unterscheiden sich von Männern beispielsweise in den relativen Anteilen bestimmter Bakterienarten wie Catenibacterium (Korrelation mit Inflammation) und Kandleria (Korrelation mit dem Androgenspiegel), wohingegen Frauen mit PCOS ähnliche Anteile von Keimen in der Darmflora wie Männer aufweisen [20].

Prinzipiell hat die Darmschleimhaut bei Frauen im Vergleich zu Männern eine dickere Mukusschicht, was mit einer verbesserten Darmbarriere einhergeht. Damit verbunden sind ein verminderter Einstrom von Endotoxinen und eine geringere inflammatorische Antwort des Immunsystems. Dies führt wiederum zu einer erhöhten Glukosetoleranz sowie zum Schutz vor kardiovaskulären Erkrankungen und zu einer tendenziell verzögerten Entwicklung eines Typ 2 Diabetes mellitus [21] - Erkrankungen, die in einem engen pathophysiologischen Zusammenhang mit der Entwicklung des PCOS stehen. Bei Frauen mit PCOS sind die Zonulinkonzentrationen im Serum erhöht, dies weist auf eine Störung der "tight junctions" ("leaky gut") hin. Dadurch kommt es zum Einstrom von LPS und zur Entwicklung einer Insulinresistenz [22].

\section{Verminderte Diversität des Mikrobioms bei PCOS}

Zur Beschreibung der Diversität des Mikrobioms wird der Shannon-Index (aDiversität) herangezogen. Er beschreibt die „Vielfalt" der Mikroorganismen in einem Habitat. Im Vergleich zu Frauen ohne Adipositas und ohne PCOS ist bei Frauen mit PCOS und Adipositas der ShannonIndex, d. h. die Artenvielfalt der Darmflora, signifikant reduziert $(p<0,01)$. Dabei fällt auf, dass die relativen Anteile von Bacteroides, Escherichia, Blautia, Parabacteroides, Weissella und Granulicatella erhöht sind, während andere Arten mit positiven Auswirkungen für die Steuerung des Stoffwechsels (Akkermansia, Alistipes, Coprococcus, Ruminococcus) in deutlich reduzierter Zahl nachweisbar sind [23].

Diese verminderte $a$-Diversität beim PCOS korreliert signifikant $(p<0,05)$ mit einem erhöhten Testosteronspiegel [24]. Ein erhöhter Testosteronspiegel wiederum korreliert positiv mit den Serumspiegeln von hs-CRP (",high sensitivity CRP“) und LBP (Lipopolysaccharid-bindendes Protein als Marker eines Endotoxineinstroms; [16]). Daraus resultiert eine „silent inflammation" (- Abb. 1).

\section{॥ Eine verminderte $a$-Diversität im Darmmikrobiom korrelierte mit einem erhöhten Testosteronspiegel}

Diese chronisch inflammatorische Reaktion ist neben erhöhten Testosteronspiegeln auch durch verminderte Östrogenspiegel sowie eine reduzierte Bildung kurzkettiger Fettsäuren (,short-chain fatty acids", SCFA), wie etwa Butyrat, durch intestinale Bakterienarten bei Frauen mit PCOS erklärbar [25]. Durch einen Mangel an Butyrat sinkt die Produktion von IL-10 (Interleukin-10) durch Treg-Zellen (regulatorische T-Zellen) was dazu führt, dass vermehrt proinflammatorische Interleukine, z. B. IL-17A, durch Th17-Zellen (T-Helferzellen) freigesetzt werden [26-28]. Diese erhöhten IL-17A-Spiegel korrelieren positiv mit der Konzentration von Trimethylamin$\mathrm{N}-$ Oxid (TMAO) im Blut [29].

\section{Insulinresistenz und Androgene}

In Zusammenhang mit dem PCOS ist die Entwicklung einer Insulinresistenz mit erhöhten Insulinspiegeln von Bedeutung. Chronisch erhöhte Insulin- und IGF-1Spiegel (,insulin like growth factor $1^{\prime \prime}$ ) steigern die Androgensynthese.

Darüber hinaus stimuliert TNF- $a$ in vitro die Proliferation von Thekazellen mit LH(luteinisierendes Hormon)-abhängiger Produktion von Androstendion und Testosteron [30]. Gleichzeitig vermindert Insulin die Synthese von SHBG in der Leber, woraus eine Zunahme der bioverfügbaren Androgene resultiert [30].

\section{Gallensäurenstoffwechsel}

Bei Frauen mit PCOS finden sich hohe Anteile von Bacteroides vulgatus bei gleichzeitig verminderten Konzentrationen an sekundären Gallensäuren (Tauro- bzw. Glycin-Desoxycholsäure) im Darm. Sekundäre Gallensäuren binden an den nukleären Far- 
nesoid-X-Faktor (FXR) sowie an den membrangebundenen "Takeda G-protein coupled receptor 5“ (TGR5) der L-Zellen des Darms. Damit steuern sie zusammen mit SCFA die Freisetzung von GLP-1 ("glucagon-like peptide 1“; [31]).

Der Mangel an sekundären Gallensäuren trägt damit zur Entwicklung eines Typ 2 Diabetes mellitus bei.

\section{Rolle des Trimethylamin-N-Oxid (TMAO)}

TMAO entsteht in der Leber aus Trimethylamin (TMA), ein Molekül, das im Kolon durch Abbau beispielsweise von Carnitin durch Bakterien entsteht [32-35]. Insbesondere Bakterienarten wie Escherichia coli, Klebsiella species (spp.), Citrobacter spp., Enterobacter spp. und andere Bakterienarten, deren Zellwand hohe Anteile an Lipopolysacchariden (Endotoxin) aufweisen, sind daran beteiligt $[36,37]$.

Erhöhte TMAO-Spiegel korrelieren mit einer gesteigerten Thrombozytenaggregation, Erkrankungen des Herz-KreislaufSystems über atherosklerotische Veränderungen der Blutgefäße, Typ 2 Diabetes mellitus und Einschränkungen der Nierenfunktion [32-35, 38]. Bei Frauen mit PCOS werden höhere Serumspiegel TMAO, sowie eine positive Korrelation zwischen TMAO und Testosteron gefunden [29, 39]. TMAO-Spiegel beeinflussen somit beim PCOS nicht nur die Risiken für kardiovaskuläre Erkrankungen und Typ 2 Diabetes mellitus, sondern auch die Höhe der Testosteronkonzentration.

Die Höhe der TMAO-Konzentrationen ist u. a. von der Ernährungsform abhängig: ein hoher Anteil an Cholin, Carnitin und Betain, gesättigten Fettsäuren (33-42\% der zugeführten Energie) sowie Getränke mit hohem Gehalt an Fructose (,Western diet") führen zu relativ hohen TMAOSpiegeln. Dies verändert im Vergleich zu einer ballaststoffreichen Ernährungsform die Zusammensetzung der Darmflora dahingehend, dass die Anteile an Proteobakterien stark zunehmen, also Arten, die sowohl TMA produzieren als auch Endotoxin freisetzen [40].
Hier steht eine Anzeige.

Springer 


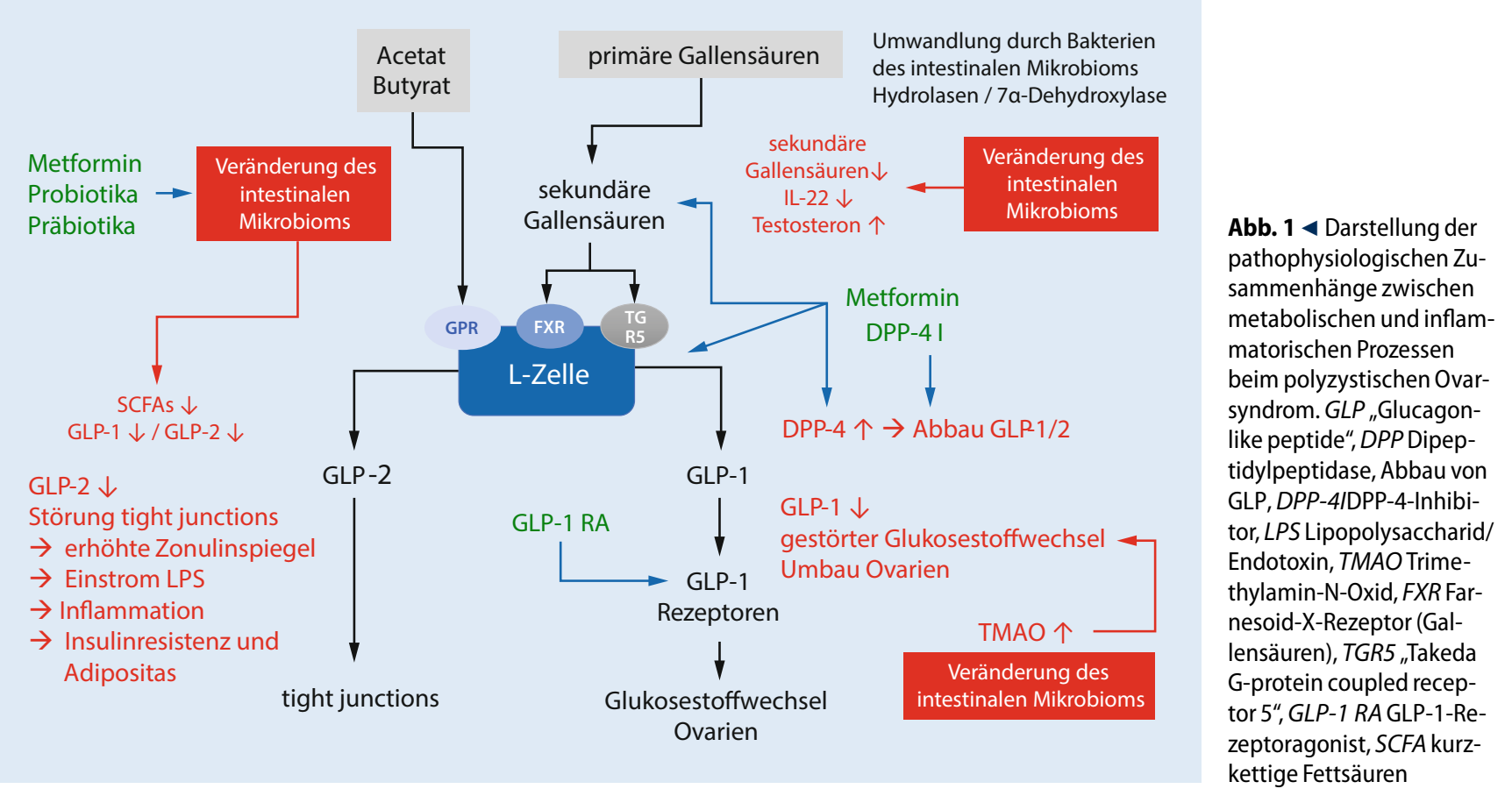

\section{Therapieoptionen}

Aus dem oben Gesagten ergibt sich ein komplexer Zusammenhang zwischen dem intestinalen Mikrobiom und dem PCOS. Diese Erkenntnisse tragen zum Verständnis der pathophysiologischen Zusammenhänge bei. Gleichzeitig eröffnet dies interessante Therapiemöglichkeiten. Neben den bekannten Optionen zur endokrinologischen Intervention (Gabe von Antiandrogenen, Zyklusregulation durch kombinierte orale Kontrazeptiva [KOK], ovarielle Stimulation mit Letrozol etc.) besteht die Möglichkeit der therapeutischen Intervention zum Beispiel durch Veränderung des Ernährungs- und Lebensstils mit nachfolgender Gewichtsreduktion.

\section{Gewichtsreduktion}

Es besteht ein positiver Zusammenhang zwischen dem Anteil an Körperfett und chronisch inflammatorischen Prozessen [41, 42]. Rezente Studien zeigen, dass durch Gewichtsabnahme die Darmwandpermeabilität signifikant abnimmt, begleitet von einer Senkung des Nüchternblutzuckers und einem Rückgang von Entzündungsparametern (IL-6) [43]. Gleichzeitig mit der Gewichtsreduktion erhöhen sich die relativen Anteile von Akkermansia muciniphila, einer Bakterienart, der ei- ne wichtige Rolle bei der Steuerung der Durchlässigkeit der Darmwand sowie des Glukosestoffwechsels zukommt [44]. Je niedriger der Anteil an A. muciniphila im intestinalen Mikrobiom ist, desto höher ist die Durchlässigkeit mit Einstrom von Endotoxin und nachfolgender Induktion einer niederschwelligen chronischen Inflammation. Die durch Gewichtsreduktion erzielte Zunahme des Anteils von A. muciniphila führt somit zur Abnahme der Darmwandpermeabilität und damit zu einer Verminderung des Einstroms von Endotoxinen in den Kreislauf [45].

Gleichzeitig korreliert A. muciniphila positiv mit der Anzahl der L-Zellen mit Produktion von GLP-1, das neben anderen Eigenschaften einen entscheidenden Einfluss auf den Glukosestoffwechsel hat [46].

Bei Frauen mit PCOS sind die Serumkonzentrationen von GLP-1 auch durch erhöhte Spiegel von Dipeptidylpetidase-4 (Abbau von GLP-1) typischerweise erniedrigt. Erste Daten weisen darauf hin, dass die Gabe von GLP-1 bei Frauen mit PCOS zu einer Verbesserung der Zyklizität und damit auch der Fertilität führt [47, 48].

Eine Restriktion der Nahrungsaufnahme auf den Zeitraum zwischen 8 und $16 \mathrm{Uhr}$ (Intervallfasten) über 6 Wochen bei übergewichtigen Frauen mit PCOS kann zu einer signifikanten Reduktion des BMI, des
hs-CRP, des HoMA-Index und des Gesamttestosterons sowie zu einer Zunahme des SHBGs führen [49].

\section{Metformin}

Metformin, ein Biguanidderivat, wird seit vielen Jahren in der Therapie des Typ 2 Diabetes mellitus, aber auch bei Frauen mit PCOS und Insulinresistenz eingesetzt (CAVE „off-label-use“; [50]).

Die Wirksamkeit von Metformin ist nur bei oraler Gabe gegeben. Metformin führt im Kolon zu einer positiven Veränderung des Mikrobioms, z.B. zu einer Zunahme des Anteils von A. muciniphila [51-53]. Darüber hinaus wird durch Metformin der Abbau von GLP-1 inhibiert. Zusätzlich werden die hepatische Glukoneogenese sowie das hepatische Fettgewebe reduziert.

Im Mausmodell resultiert die Gabe von Metformin in einem signifikanten Abfall des Testosteronspiegels sowie einem Rückgang des Endotoxinspiegels. Gleichzeitig zeigen sich auch Veränderungen des intestinalen Mikrobioms mit Abnahme der Anteile von Proteobakterien (Endotoxin) und der Zunahme von Bifidobakterien [54]. Bei Frauen mit PCOS und Übergewicht resultiert nach den Ergebnissen einer Metaanalyse die Einnahme von Metformin in einer Verminderung des BMI, des Taillenumfangs, der FSH(follikelstimulierendes Hor- 
mon)- und LH-Spiegel, des Testosterons sowie des LDL(„Iow-density lipoproteins“)Cholesterins [55].

Klinisch verbessern sich durch Metformin die Zyklizität sowie die Ovulationsrate. Ebenso ist das Ansprechen auf eine ovarielle Stimulation (bei Kinderwunsch) besser [56, 57]. Darüber hinaus lässt sich durch Metformingabe das Risiko zur Entwicklung eines ovariellen Überstimulationssyndroms im Zusammenhang mit einer ovariellen Stimulation deutlich reduzieren.

\section{Ballaststoffe}

Die Ernährung bei Frauen mit PCOS spielt in der Therapie eine entscheidende Rolle. Untersuchungen bei Ratten weisen auf den negativen Einfluss einer Ernährung mit hohen Anteilen raffinierter Kohlenhydrate auf den Reproduktionstrakt mit der Entwicklung PCOS-ähnlicher Veränderungen hin [58].

Tatsächlich scheint nach einer Metaanalyse eine Diät mit einem niedrigen glykämischen Index bei PCOS von Vorteil zu sein: Absenkung des HoMA-Indexes, Verminderung der Nüchternblutglukose, des Gesamtcholesterins, des LDL-Cholesterins sowie der Triglyzeride, Reduktion des Taillenumfangs sowie des Gesamttestosterons (alle $p<0,05$; [59]).

In der „praktischen Anwendung" lässt sich nachweisen, dass beispielsweise im Vergleich zu einem Abendessen mit Weißbrot der Verzehr von Vollkornbrot mit einer signifikanten Zunahme des GLP-1-Spiegels korreliert [60]. Ebenso führt die Umstellung der Ernährungsform auf eine mediterrane Diät (kaltgepresstes Olivenöl, Hülsenfrüchte, Nüsse, Vollkornprodukte, Gemüse und Früchte, Fisch, mäßiger Genuss von Rotwein und Verminderung des Fleischkonsums) bei Frauen mit PCOS zu einer Reduktion des CRP-Spiegels sowie des HoMAIndexes [61, 62].

\section{Probiotika}

Bei Frauen mit PCOS führt die Gabe eines Probiotikums mit dem Stamm Bifidobacterium lactis V9 zu erhöhten Konzentrationen von SCFA sowie zu einer Steigerung der Freisetzung von PYY (Peptid YY) und Ghrelin, welche über die Darm-Hirn-Ach- se in einer Abnahme von $\mathrm{LH}$ sowie des LH/FSH-Quotienten resultiert [25].

Positive Wirkungen eines weiteren Probiotikums aus verschiedenen Bakterienstämmen (Lactobacillus acidophilus, L. reuteri, L. fermentum, Bifidobacterium bifidum) plus $200 \mu \mathrm{g} / \mathrm{Tag}$ Selen waren bei Gabe über 12 Wochen im Vergleich zur Kontrollgruppe zu sehen: eine signifikante Verbesserung des Hirsutismus sowie eine signifikante Abnahme von hs-CRP- und Testosteronspiegeln [63].

Es handelt sich hierbei sicherlich um vorläufige Daten, jedoch lassen die Studienergebnisse erkennen, dass es durch Veränderung des Darmmikrobioms möglich erscheint, einen klinisch bedeutsamen Einfluss auf endokrine Prozesse zu nehmen, die zur Entstehung und Ausprägung des PCOS beitragen.

\section{Präbiotika und andere Nahrungs- ergänzungsmittel}

Im Tierversuch mit PCOS-Ratten reduziert Berberin eine Insulinresistenz und senkt den Testosteronspiegel [64]. In einer Metaanalyse von klinischen Studien scheint Berberin einer Metformingabe bei der Verminderung einer Insulinresistenz, der Verbesserung einer Dyslipidämie sowie der Senkung des Testosteronspiegels ebenbürtig zu sein [65].

Auch die Gabe von $2 \times 500 \mathrm{mg} / \mathrm{Tag}$ Quercetin bei PCOS hat einen positiven Effekt: über die Zunahme der Expression von Adiponectin-Rezeptoren, was zu einer Beeinflussung der Insulinsensitivität führt [66].

Eine Nahrungsergänzung mit Phytoöstrogenen (Isoflavon) in Form von Genistein $(2 \times 18 \mathrm{mg} / \mathrm{Tag})$ resultiert nach 3 Monaten bei PCOS in einer Reduktion der LH-, Testosteron- und der DHEAS(Dehydroepiandrosteronsulfat)-Spiegel [67].

Die Einnahme von $50 \mathrm{mg} / \mathrm{Tag}$ Sojaisoflavonen über 12 Wochen führte bei Frauen mit PCOS zur Reduktion der Insulinspiegel und des HoMA-Indexes sowie zu einer Verminderung des FAI und der Serumtriglyzeride [68].

Ein ähnlicher Effekt bei PCOS wurde bei der täglichen Gabe von $200 \mu \mathrm{g}$ Chrom (als Chrompicolinat) über 8 Wochen auf den Insulinspiegel sowie den HoMA-Index zusammen mit einer Reduktion des hs-CRP-
Hier steht eine Anzeige.

Springer 
Spiegels sowie einer günstigen Auswirkung auf Akne und Hirsutismus beobachtet $[68,69]$.

Der regelmäßige Verzehr von Lakritze verringert bei gesunden Frauen den Testosteronspiegel und kann als adjuvante Therapie bei Hirsutismus und PCOS in Erwägung gezogen werden [70].

In Tierversuchen zeigen sich bei der Gabe von Inulin bzw. Resveratrol (letzteres eventuell auch in Kombination mit Metformin) positive Effekte mit Besserung der ovariellen Histologie bzw. einer Suppression der inflammatorischen Marker [54, 71].

\section{Fazit für die Praxis}

- Das PCOS (Syndrom polyzystischer Ovarien) gehört zu den häufigsten Endokrinopathien überhaupt, die weltweite Prävalenz wird auf 5-12\% geschätzt, die Ursache ist bis heute unbekannt.

- Der klinische Phänotyp zeigt eine hohe Variabilität, entsprechend unterschiedlich sind auch die Therapiekonzepte.

- Neben den endokrinologischen Aspekten rücken zunehmend die für das PCOS charakteristischen metabolischen Veränderungen in den Fokus des Interesses.

- Veränderungen des Darmmikrobioms haben einen Einfluss auf die Entstehung und den klinischen Verlauf des PCOS.

- Daraus ergeben sich sowohl diagnostische Hinweise als auch neue Therapieoptionen: So wirken sich eine Gewichtsreduktion, der Einsatz von Metformin, eine Modifikation der Ernährungsform sowie Prä- und Probiotika auf die Zusammensetzung des Darmmikrobioms und darüber auf Ausprägung und Verlauf des PCOS aus.

- Zu erwarten ist, dass die Analyse des Darmmikrobioms in Zukunft zu den empfohlenen diagnostischen Verfahren zählen wird, um daraus die o.g. therapeutischen Maßnahmen abzuleiten.

Korrespondenzadresse

Prof. Dr. Christoph Keck

Medical Affairs, Amedes MDL

Haferweg 36, 22769 Hamburg, Deutschland

Christoph.keck@amedes-group.com

\section{Einhaltung ethischer Richtlinien}

Interessenkonflikt. W.R. Heizmann und C. Keck geben an, dass kein Interessenkonflikt besteht.

Für diesen Beitrag wurden von den Autoren keine Studien an Menschen oder Tieren durchgeführt.
Für die aufgeführten Studien gelten die jeweils dort angegebenen ethischen Richtlinien.

Open Access. Dieser Artikel wird unter der Creative Commons Namensnennung 4.0 International Lizenz veröffentlicht, welche die Nutzung, Vervielfältigung, Bearbeitung, Verbreitung und Wiedergabe in jeglichem Medium und Format erlaubt, sofern Sie den/die ursprünglichen Autor(en) und die Quelle ordnungsgemäß nennen, einen Link zur Creative Commons Lizenz beifügen und angeben, ob Änderungen vorgenommen wurden.

Die in diesem Artikel enthaltenen Bilder und sonstiges Drittmaterial unterliegen ebenfalls der genannten Creative Commons Lizenz, sofern sich aus der Abbildungslegende nichts anderes ergibt. Sofern das betreffende Material nicht unter der genannten Creative Commons Lizenz steht und die betreffende Handlung nicht nach gesetzlichen Vorschriften erlaubt ist, ist für die oben aufgeführten Weiterverwendungen des Materials die Einwilligung des jeweiligen Rechteinhabers einzuholen.

Weitere Details zur Lizenz entnehmen Sie bitte der Lizenzinformation auf http://creativecommons.org/ licenses/by/4.0/deed.de.

\section{Literatur}

1. Ndefo UA, Eaton A, Green MR (2013) Polycystic ovary syndrome: a review of treatment options with a focus on pharmacological approaches. P T 38(6):336-355

2. WolfWM, WattickRA, Kinkade ON, OlfertMD(2018) Geographical prevalence of polycystic ovary syndrome as determined by region and race/ethnicity. Int JEnviron Res Public Health 15(11):2589. https:// doi.org/10.3390/ijerph15112589

3. Amiri M, Ramezani Tehrani F, Nahidi F, Bidhendi Yarandi R, Behboudi-Gandevani S, Azizi F (2017) Association between biochemical hyperandrogenism parameters and Ferriman-Gallwey score in patients with polycystic ovary syndrome: a systematic review and meta-regression analysis. Clin Endocrinol (Oxf) 87(3):217-230. https://doi. org/10.1111/cen.13389

4. Alexiou E et al (2017) Hyperandrogenemia in women with polycystic ovary syndrome: prevalence, characteristics and association with body mass index. Horm Mol Biol Clin Investig 29(3):105-111

5. Bozdag G et al (2016) The prevalence and phenotypic features of polycystic ovary syndrome: a systematic review and meta-analysis. Hum Reprod 31(12):2841-2855

6. Teede HJ et al (2018) Recommendations from the international evidence-based guideline for the assessment and management of polycystic ovary syndrome. Hum Reprod 33(9):1602-1618

7. Vermeulen A, Verdonck L, Kaufman JM (1999) A critical evaluation of simple methods for the estimation of free testosterone in serum. J Clin Endocrinol Metab 84(10):3666-3672

8. Legro RS et al (1999) Prevalence and predictors of risk for type 2 diabetes mellitus and impaired glucose tolerance in polycystic ovary syndrome: a prospective, controlled study in 254 affected women. JClin Endocrinol Metab 84(1):165-169

9. Gupta M, Yadav R, MaheyR, Agrawal A, UpadhyayA, Malhotra N, Bhatla N (2019) Correlation of body mass index (BMI), anti-mullerian hormone (AMH), and insulin resistance among different polycystic ovary syndrome (PCOS) phenotypes-a cross-sectional study. Gynecol Endocrinol 35(11):970-973. https://doi.org/10.1080/09513590.2019.1613640

10. Sender R, Fuchs S, Milo R (2016) Revised estimates for the number of human and bacteria cells in the body. PLoS Biol 14(8):e1002533. https://doi.org/ 10.1371/journal.pbio. 1002533

11. Cani PD (2018) Human gut microbiome: hopes, threats and promises. Gut 67(9):1716-1725. https://doi.org/10.1136/gutjnl-2018-316723

12. Yang $X$, Xie L, Li Y, Wei C (2009) More than $9,000,000$ unique genes in human gut bacterial community: estimating gene numbers inside a human body. PLoS One 4(6):e6074. https://doi.org/10.1371/ journal.pone.0006074

13. Grice EA, Segre JA (2012) The human microbiome: our second genome. Annu Rev Genomics Hum Genet 13:151-170. https://doi.org/10.1146/ annurev-genom-090711-163814

14. Tremellen K, Pearce K (2012) Dysbiosis of Gut Microbiota (DOGMA)-a novel theory for the development of Polycystic Ovarian Syndrome. Med Hypotheses 79(1):104-12. https://doi.org/10. 1016/j.mehy.2012.04.016

15. González F (2012) Inflammation in polycysticovary syndrome: underpinning of insulin resistance and ovarian dysfunction. Steroids 77(4):300-305. https://doi.org/10.1016/j.steroids.2011.12.003

16. Zhu Q, Zhou H, Zhang A, Gao R, Yang S, Zhao C, Wang Y, Hu J, Goswami R, Gong L, Li Q (2016) Serum LBP is associated with insulin resistance in women with PCOS. PLoS ONE 11(1):e145337. https://doi. org/10.1371/journal.pone.0145337

17. Elderman M, de Vos P, Faas M (2018) Role of microbiota in sexually dimorphic immunity. Front Immunol 9:1018. https://doi.org/10.3389/fimmu. 2018.01018

18. Kim YS, Unno T, Kim BY, Park MS (2020) Sex differences in gut microbiota. World J Mens Health 38(1):48-60. https://doi.org/10.5534/ wjmh.190009

19. Shin JH, Park YH, Sim M, Kim SA, Joung H, Shin DM (2019) Serum level of sex steroid hormone is associated with diversity and profiles of human gut microbiome. Res Microbiol 170(4-5):192-201. https://doi.org/10.1016/j.resmic.2019.03.003

20. Insenser M, Murri M, Del Campo R, Martínez-García MÁ, Fernández-Durán E, Escobar-Morreale HF (2018)Gutmicrobiota and the polycysticovarysyndrome: influence of sex, sexhormones, and obesity. JClinEndocrinol Metab 103(7):2552-2562. https:// doi.org/10.1210/jc.2017-02799

21. Cross TL, Kasahara K, Rey FE (2018) Sexual dimorphism of cardiometabolic dysfunction: gut microbiome in the play? Mol Metab 15:70-81. https://doi.org/10.1016/j.molmet.2018.05.016

22. Zhang D, Zhang L, Yue F, Zheng Y, Russell $R$ (2015) Serum zonulin is elevated in women with polycystic ovary syndrome and correlates with insulin resistance and severity of anovulation. Eur J Endocrinol 172(1):29-36. https://doi.org/10.1530/ EJE-14-0589

23. Liu R, Zhang C, Shi Y, Zhang F, Li L, Wang X, Ling Y, Fu H, Dong W, Shen J, Reeves A, Greenberg AS, Zhao L, Peng Y, Ding X (2017) Dysbiosis of gut microbiota associated with clinical parameters in polycystic ovary syndrome. Front Microbiol 8:324. https://doi.org/10.3389/fmicb.2017.00324

24. Torres PJ, Siakowska M, Banaszewska B, Pawelczyk L, Duleba AJ, Kelley ST, Thackray VG (2018) Gut microbial diversity in women with polycystic ovary syndrome correlates with hyperandroge- 
nism. J Clin Endocrinol Metab 103(4):1502-1511. https://doi.org/10.1210/jc.2017-02153

25. Zhang J, Sun Z, Jiang S, Bai X, Ma C, Peng Q, Chen K, Chang H, Fang T, Zhang H (2019) Probiotic bifidobacterium lactis $\mathrm{V} 9$ regulates the secretion of sex hormones in polycystic ovary syndrome patients through the gut-brain axis. mSystems 4(2):e17-19. https://doi.org/10.1128/mSystems. 00017-19

26. Brunsing RL, Prossnitz ER (2011) Induction of interleukin-10 in the Thelper type 17 effector population by the $G$ protein coupled estrogen receptor (GPER) agonist G-1. Immunology 134(1):93-106. https://doi.org/10.1111/j.13652567.2011.03471.x

27. Cady N, Peterson SR, Freedman SN, Mangalam AK (2020) Beyond metabolism: the complex interplay between dietary phytoestrogens, gutbacteria, and cells of nervous and immune systems. Front Neurol 11:150. https://doi.org/10.3389/fneur.2020.00150

28. Rooks MG, Garrett WS (2016) Gut microbiota, metabolites and host immunity. Nat Rev Immunol 16(6):341-352. https://doi.org/10.1038/nri.2016. 42

29. Huang J, Liu L, Chen C, Gao Y (2020) PCOS without hyperandrogenism is associated with higher plasma trimethylamine $\mathrm{N}$-oxide levels. BMC Endocr Disord 20(1):3. https://doi.org/10.1186/ s12902-019-0486-9

30. Nestler JE, Powers LP, Matt DW, Steingold KA, Plymate SR, Rittmaster RS, Clore JN, Blackard WG (1991) A direct effect of hyperinsulinemia on serum sex hormone-binding globulin levels in obese women with the polycystic ovary syndrome. J Clin Endocrinol Metab 72(1):83-89. https://doi. org/10.1210/jcem-72-1-83

31. Xie C, Huang W, Young RL, Jones KL, Horowitz M, Rayner CK, Wu T (2021) Role of bile acids in the regulation of food intake, and their dysregulation in metabolic disease. Nutrients 13(4):1104. https:// doi.org/10.3390/nu13041104

32. Wang Z, Klipfell E, Bennett BJ, Koeth R, Levison BS, Dugar B, Feldstein AE, Britt EB, Fu X, Chung YM, Wu Y, Schauer P, Smith JD, Allayee $H$, Tang WH, DiDonato JA, Lusis AJ, Hazen SL (2011) Gut flora metabolism of phosphatidylcholine promotes cardiovascular disease. Nature 472(7341):57-63. https://doi.org/10.1038/nature09922

33. Koeth RA et al (2013) Intestinal microbiota metabolism of L-carnitine, a nutrient in red meat, promotes atherosclerosis. Nat Med 19(5):576-585. https://doi.org/10.1038/nm.3145

34. Ufnal M, Jazwiec R, Dadlez M, Drapala A, Sikora M, Skrzypecki J (2014) Trimethylamine-N-oxide: a carnitine-derived metabolite that prolongs the hypertensive effect of angiotensin II in rats. Can J Cardiol 30(12):1700-1705. https://doi.org/10. 1016/j.cjca.2014.09.010

35. Hayashi T, Yamashita T, Watanabe H, Kami K, Yoshida N, Tabata T, Emoto T, Sasaki N, Mizoguchi T, Irino $\mathrm{Y}$, Toh R, Shinohara M, Okada Y, Ogawa W, Yamada T, Hirata KI (2018) Gut microbiome and plasma microbiome-related metabolites in patients with decompensated and compensated heart failure. Circ J 83(1):182-192. https://doi.org/ 10.1253/circj.CJ-18-0468

36. Rath S, Heidrich B, Pieper DH, Vital M (2017) Uncovering the trimethylamine-producing bacteria of the human gut microbiota. Microbiome 5(1):54 https://doi.org/10.1186/s40168-017-0271-9

37. Al-Obaide MAl, Singh $R$, Datta $P$, Rewers-Felkins KA, Salguero MV, Al-Obaidi I, Kottapalli KR, Vasylyeva TL (2017) Gut microbiota-dependent trimethylamine- $\mathrm{N}$-oxide and serum biomarkers in patients with T2DM and advanced CKD. J Clin Med 6(9):86. https://doi.org/10.3390/jcm6090086

38. Janeiro MH, Ramírez MJ, Milagro Fl, Martínez JA, Solas M (2018) Implication of trimethylamine $\mathrm{N}$-oxide (TMAO) in disease: potential biomarker or new therapeutic target. Nutrients 10(10):1398 https://doi.org/10.3390/nu10101398

39. Eyupoglu ND, Caliskan Guzelce E, Acikgoz A, Uyanik E, Bjørndal B, Berge RK, Svardal A, Yildiz BO (2019) Circulating gut microbiota metabolite trimethylamine $\mathrm{N}$-oxide and oral contraceptive use in polycystic ovary syndrome. Clin Endocrinol (Oxf) 91(6):810-815. https://doi.org/10.1111/cen. 14101

40. Simpson HL, Campbell BJ (2015) Review article: dietary fibre-microbiota interactions. Aliment Pharmacol Ther 42(2):158-179. https://doi.org/ 10.1111/apt.13248

41. Martínez I, Lattimer JM, Hubach KL, Case JA, Yang J, Weber CG, Louk JA, Rose DJ, Kyureghian G Peterson DA, Haub MD, Walter J (2013) Gut microbiome composition is linked to whole grain-induced immunological improvements. ISME J 7(2):269-280. https://doi.org/10.1038/ ismej.2012.104

42. Hestiantoro A et al (2018) Body fat percentage is a better marker than body mass index for determining inflammation status in polycystic ovary syndrome. Int J Reprod Biomed 16(10):623-628

43. Damms-Machado A, Louis S, Schnitzer A, Volynets V, Rings A, Basrai M, Bischoff SC (2017) Gut permeability is related to body weight, fatty liver disease, and insulin resistance in obese individuals undergoing weight reduction. Am J Clin Nutr 105(1):127-135. https://doi.org/10.3945/ajcn. 116.131110

44. Louis S, Tappu RM, Damms-Machado A, Huson DH, Bischoff SC (2016) Characterization of the gut microbial community of obese patients following a weight-loss intervention using whole metagenome shotgun sequencing. PLoS ONE 11(2):e149564. https://doi.org/10.1371/journal. pone. 0149564

45. Everard A, Belzer C, Geurts L, Ouwerkerk JP, DruartC,BindelsLB, GuiotY,Derrien M, MuccioliGG, DelzenneNM, de VosWM, Cani PD (2013) Cross-talk between Akkermansia muciniphila and intestinal epithelium controls diet-induced obesity. Proc Natl Acad SciU SA 110(22):9066-9071.https://doi.org/ 10.1073/pnas.1219451110

46. Li X, Watanabe K, Kimura I (2017) Gut microbiota dysbiosis drives and implies novel therapeutic strategies for diabetes mellitus and related metabolic diseases. Front Immunol 8:1882. https:// doi.org/10.3389/fimmu.2017.01882

47. Jensterle M, Janez A, Fliers E, DeVries JH, VrtacnikBokal E, Siegelaar SE (2019) The role of glucagonlike peptide- 1 in reproduction: from physiology to therapeutic perspective. Hum Reprod Update 25(4):504-517.https://doi.org/10.1093/humupd/ dmz019

48. Öztürk B, Gürbüz AS, Durak ZE, Öztürk HS (2018) Dipeptidyl peptidase- 4 and adenosine deaminase enzyme levels in polycystic ovary syndrome. Gynecol Endocrinol 35(2):138-141. https://doi. org/10.1080/09513590.2018.1505847

49. Li C, Xing C, Zhang J, Zhao H, Shi W, He B (2021) Eight-hour time-restricted feeding improves endocrine and metabolic profiles in women with anovulatory polycystic ovary syndrome. J Transl Med 19(1):148. https://doi.org/10.1186/ s12967-021-02817-2

50. Apovian CM, Aronne LJ, Bessesen DH, McDonnell ME, Murad MH, Pagotto U, Ryan DH,
Still CD, Endocrine Society (2015) Pharmacological management of obesity: an endocrine Society clinical practice guideline. J Clin Endocrinol Metab 100(2):342-362. https://doi.org/10.1210/jc.20143415

51. LeeH, Ko G (2014) Effect of metformin on metabolic improvement and gut microbiota. Appl Environ Microbiol 80(19):5935-5943. https://doi.org/10. 1128/AEM.01357-14

52. de la Cuesta-Zuluaga J, Mueller NT, CorralesAgudelo V, Velásquez-Mejía EP, Carmona JA, Abad JM, Escobar JS (2017) Metformin is associated with higher relative abundance of mucindegrading Akkermansia muciniphila and several short-chain fatty acid-producing microbiota in the gut. Diabetes Care 40(1):54-62. https://doi.org/10. 2337/dc16-1324

53. Minamii T, Nogami M, Ogawa W (2018) Mechanisms of metformin action: In and out of the gut. J Diabetes Investig 9(4):701-703. https://doi.org/ 10.1111/jdi.12864

54. Xue J, Li X, Liu P, Li K, Sha L, Yang X, Zhu L, Wang Z, Dong $Y$, Zhang L, Lei H, Zhang X, Dong X, Wang $H$ (2019) Inulin and metformin ameliorate polycystic ovary syndrome via anti-inflammation and modulating gut microbiota in mice. Endocr J 66(10):859-870. https://doi.org/10.1507/endocrj. EJ18-0567

55. Guan Y, Wang D, Bu H, Zhao T, Wang H (2020) The effect of metformin on polycystic ovary syndrome in overweight women: a systematic review and meta-analysis of randomized controlled trials. Int J Endocrinol 2020:5150684. https://doi.org/10. 1155/2020/5150684

56. Di Pietro M, Velazquez C, Matzkin ME, Frungieri MB, Peña MG, de Zúñiga I, Pascuali N, Irusta G, Bianchi MS, Parborell F, Abramovich D (2020) Metformin has a direct effect on ovarian cells that is dependent on organic cation transporters. Mol Cell Endocrinol 499:110591. https://doi.org/10.1016/j. mce.2019.110591

57. Tejpal C, Poudel I, Jahan N (2019) Is metformin the answer for distressed females with menstrual irregularities? Cureus 11(8):e5460.https://doi.org/ 10.7759/cureus.5460

58. Niño OMS, da Costa CS, Torres KM, Zanol JF, Freitas-Lima LC, Miranda-Alves L, Graceli JB (2020) High-refined carbohydrate diet leads to polycystic ovary syndrome-like features and reduced ovarian reserve in female rats. Toxicol Lett 332:42-55. https://doi.org/10.1016/j.toxlet.2020.07.002

59. Kazemi M, Hadi A, Pierson RA, Lujan ME, Zello GA, Chilibeck PD (2020) Effects of dietary glycemic index and glycemic load on cardiometabolic and reproductive profiles in women with polycystic ovary syndrome: a systematic review and metaanalysis of randomized controlled trials. Adv Nutr 12(1):161-178. https://doi.org/10.1093/ advances/nmaa092

60. Nilsson AC, Ostman EM, Holst JJ, Björck IM (2008) Including indigestible carbohydrates in the evening meal of healthy subjects improves glucose tolerance, lowers inflammatory markers, and increases satiety after a subsequent standardized breakfast. J Nutr 138(4):732-739. https://doi.org/ 10.1093/jn/138.4.732

61. Widmer RJ, Flammer AJ, Lerman LO, Lerman A (2015) The Mediterranean diet, its components, and cardiovascular disease. Am J Med 128(3):229-238. https://doi.org/10.1016/j. amjmed.2014.10.014

62. Barrea L, Arnone A, Annunziata G, Muscogiuri G, Laudisio D, Salzano C, Pugliese G, Colao A, Savastano S (2019) Adherence to the Mediterranean 
diet, dietary patterns and body composition in women with polycystic ovary syndrome (PCOS). Nutrients 11(10):2278. https://doi.org/10.3390/ nu11102278

63. Jamilian M, Mansury S, Bahmani F, Heidar Z, Amirani E, Asemi Z (2018) The effects of probiotic and selenium co-supplementation on parameters of mental health, hormonal profiles, and biomarkers of inflammation and oxidative stress in women with polycystic ovary syndrome. J Ovarian Res 11(1):80. https://doi.org/10.1186/s13048-0180457-1

64. Shen HR, Xu X, Li XL (2021) Berberine exerts a protective effect on rats with polycystic ovary syndrome byinhibiting the inflammatory response and cell apoptosis. Reprod Biol Endocrinol 19(1):3. https://doi.org/10.1186/s12958-020-00684-y

65. Li MF, Zhou XM, Li XL (2018) The effect of berberine on polycystic ovary syndrome patients with insulin resistance (PCOS-IR): a meta-analysis and systematic review. Evid Based Complement Alternat Med 2018:2532935. https://doi.org/10. 1155/2018/2532935

66. Rezvan N, Moini A, Gorgani-Firuzjaee S, Hosseinzadeh-Attar MJ (2018) Oral quercetin supplementation enhances adiponectin receptor transcript expression in polycystic ovary syndrome patients: a randomized placebo-controlled double-blind clinical trial.Cell J 19(4):627-633. https:// doi.org/10.22074/cellj.2018.4577

67. Khani B, Mehrabian F, Khalesi E, Eshraghi A (2011) Effect of soy phytoestrogen on metabolic and hormonal disturbance of women with polycystic ovary syndrome. J Res Med Sci 16(3):297-302

68. Jamilian M, BahmaniF, Siavashani MA, Mazloomi M, Asemi Z, Esmaillzadeh A (2016) The effects of chromium supplementation on endocrine profiles, biomarkers of inflammation, and oxidative stress in women with polycystic ovary syndrome: a randomized, double-blind, placebo-controlled trial. Biol Trace Elem Res 172(1):72-78. https://doi. org/10.1007/s12011-015-0570-6

69. Jamilian M, Asemi Z (2016) The effects of soy Isoflavones on metabolic status of patients with polycystic ovary syndrome. J Clin Endocrinol Metab 101(9):3386-3394. https://doi.org/10. 1210/jc.2016-1762

70. Armanini D, Mattarello MJ, Fiore C, Bonanni G, Scaroni C, Sartorato P, Palermo M (2004) Licorice reduces serum testosterone in healthy women. Steroids 69(11-12):763-766. https://doi.org/10. 1016/j.steroids.2004.09.005

71. Furat Rencber $S$, Kurnaz Ozbek S, Eraldemır $C$, Sezer Z, Kum T, Ceylan S, Guzel E (2018) Effect of resveratrol and metformin on ovarian reserve and ultrastructure in PCOS: an experimental study. J Ovarian Res 11(1):55. https://doi.org/10.1186/ s13048-018-0427-7

\section{Microbiome and metabolism in polycystic ovary syndrome (PCOS)}

Polycystic ovary syndrome (PCOS) is a common endocrine disorder in women of fertile age. The etiology of PCOS is unknown; however, recent research has contributed to a better understanding of its pathophysiological mechanisms. Women with PCOS suffer from endocrinological as well as metabolic disorders. In addition, it has been shown that the gut microbiome of affected compared with non-affected women shows remarkable differences. It can be expected that these findings will lead to new treatment options that could significantly alter the treatment of PCOS in the future.

\section{Keywords}

Gastrointestinal microbiome · Metabolic diseases · Endocrinology · Fertility · Androgens 